\title{
Non-fixation Versus Fixation of Mesh in Totally Extraperitoneal Repair of Inguinal Hernia: a Comparative Study
}

\author{
Ameet Kumar $^{1} \cdot$ Sumesh Kaistha ${ }^{1} \cdot$ Rajesh Gangavatiker ${ }^{1}$ \\ Received: 29 January 2017 / Accepted: 22 January 2018 / Published online: 7 February 2018 \\ (C) Association of Surgeons of India 2018
}

\begin{abstract}
Two major issues with laparoscopic inguinal hernia (IH) repair are recurrences and chronic groin pain (CGP). The procedure involves fixing the mesh with the tackers which is believed to increase the rate of CGP due to nerve injuries. Thus, non-fixation of mesh is being proposed but concerns remain regarding increased recurrences. We sought to look at our outcomes after we switched over to non-fixation of mesh in totally extraperitoneal repair (TEP). Retrospective review of prospectively maintained database of 171 repairs was done on 122 patients (fixation 59 and non-fixation 112) during a period of 4 years with an endeavor to complete a minimum of 1 year of clinical follow-up. The primary objective was to assess the recurrence rates and CGP and the secondary objective was to assess operative times, immediate post-op pain, incidence of urinary retention, duration of hospital stay, days taken to return to activity, and cost. The mean operative times for unilateral IH for the fixation and non-fixation groups were $41.8 \pm 11.4$ and $35.9 \pm 9.7 \mathrm{~min}$, respectively $(p=0.021)$, whereas for bilateral were $66.2 \pm 15.6$ and $55.3 \pm 14.2 \mathrm{~min}$, respectively $(p=0.018)$. The mean pain score was $3.44 \pm 1.2$ versus $3.01 \pm 1.0 ;(p=0.037)$ in the two groups, respectively. At a mean follow-up of $33.2 \pm 17.0$ and $18.7 \pm 6.2$ months, the incidence of CGP was $02(3.4 \%)$ and $3(2.7 \%)(p=1.000)$ and recurrences were $02(3.4 \%)$ in the two groups, respectively $(p=0.118)$. Non-fixation of mesh in TEP does not lead to increased recurrence though it does not decrease the incidence of chronic groin pain. Collateral advantage would be decreased operative times, lesser post-operative pain, and decreased costs.
\end{abstract}

Keywords Inguinal hernia $\cdot$ Laparoscopic repair $\cdot$ Totally extraperitoneal repair $\cdot$ Non-fixation ofmesh $\cdot$ Recurrence rates $\cdot$ Chronic groin pain

\section{Introduction}

Laparoscopic inguinal hernia surgery (LIHS) is the recommended procedure for bilateral and recurrent inguinal hernias (IH) while it is one of the options dealing with a unilateral IH [1]. Laparoscopic approach to inguinal hernia surgery started in the 1980s. Since then, many techniques of laparoscopic inguinal hernia repair had been developed. Today, only two

Sumesh Kaistha

sumeshkaishta@gmail.com

Ameet Kumar

docam@ rediffmail.com

Rajesh Gangavatiker

r_gangavatiker@yahoo.com

1 Department of GI Surgery, Surgical Division, Command Hospital Air Force, Bangalore 560007, India techniques are commonly employed - totally extraperitoneal repair (TEP) and the transabdominal preperitoneal repair (TAPP) $[2,3]$. Of these two, TEP has emerged as the favored technique [4].

The major issue with hernia surgery has been recurrence. However, now that low recurrence rates are being reported consistently, attention is being drawn to other issues like chronic groin pain (CGP) and quality of life (QOL) [5, 6]. The incidence of CGP groin following IH surgery has been reported between 0.03 and $31 \%$ [7, 8].

Improvising and refining surgical techniques is essential for the growth of surgery and to improve outcomes. CGP singularly affects the quality of life in patients undergoing IH surgery and remains a key area for surgeons to improve upon. CGP most often occurs due to nerve damage during LIHS which can be caused during dissection or fixation of mesh. Mesh fixation is usually done by laparoscopic tackers and multiple tacks were being used. Subsequently, in an attempt to reduce CGP, the number of tacks has now been 
reduce to two, one medially over the coopers ligament and another laterally at the level of anterior superior iliac spine [9]. Further, there have been attempts to completely avoid fixation of mesh. The concerns in avoiding mesh fixation are that in an attempt to reduce CGP, we may be increasing the chances of IH recurrence as non-fixation may lead to displacement of mesh. Three meta-analyses have now been published that have shown that non-fixation of mesh does not lead to increased recurrences [10-12].

Going with this body of evidence, we started non-fixation of mesh in TEP. We, in this study, review our experience with non-fixation of mesh and report its results.

\section{Methods}

This is a retrospective review of a prospectively maintained database of all patients undergoing TEP for IH at our center from January 2012 to June 2015. In the initial part of this period, till June 2013, mesh fixation with laparoscopic tackers was routinely done. After this period, in all patients, the mesh was not fixed. All patients completed a minimum of 1-year follow-up. The follow-up data was updated till June 2016.

The aim of this study is to evaluate the outcomes of mesh fixation versus non-fixation in TEP at our center over this period with a primary objective to evaluate the recurrence rates and chronic groin pain and secondary objective to assess the operative times, immediate post-op pain, incidence of urinary retention, duration of hospital stay, days taken to return to activity, and cost.

\section{Inclusion Criteria}

All adult patients with uncomplicated IH were offered TEP.

\section{Exclusion Criteria}

Patients with a large lower midline scars, a complicated hernia or unfit for general anesthesia.

\section{Operative Procedure}

Injection amoxicillin $1000 \mathrm{mg}$ and clavulanic acid $200 \mathrm{mg}$ was given intravenously as prophylaxis at the time of induction along with two additional post-operative doses. The procedure was done under general anesthesia. We did not employ urinary bladder catheterization.

\section{Technique of TEP Procedure}

A 10-mm paraumbilical port was made on the side of the hernia. In bilateral hernias, the port was made on the side of the larger sac. The rectus muscle was retracted laterally after incising the anterior rectus sheath, and a preperitoneal access was obtained to place a $10-\mathrm{mm}$ trocar for a $10-\mathrm{mm} 30^{\circ}$ telescope. Pneumo-preperitoneum was created and blunt dissection with the telescope was used to create the preperitoneal space. Two 5-mm ports were placed in the midline, one just above the symphysis pubis and the other in between the 10$\mathrm{mm}$ port and 5-mm supra-pubic port, and the entire posterior floor was dissected. Reduction of sac was attempted in all cases but in case of adhesions, sac was divided at the deep ring. Genitofemoral and lateral cutaneous nerves were identified. Fascia over these nerves was kept intact. Peritoneum was teased down, proximal to the point where vas deferens turns medially. The triangle of doom and Hasselbach's triangle were defined. After the dissection, a rolled $12 \times 15 \mathrm{~cm}$ polypropylene mesh was introduced via the $10-\mathrm{mm}$ port. The mesh was spread to cover the entire myopectineal area on the affected side. In bilateral hernia, both meshes were placed so as to overlap each other in the midline. The mesh was fixed with absorbable tackers, medially on Cooper's ligament and laterally near anterior superior iliac spine above the iliopubic tract. This step was omitted during the mesh non-fixation period. For bilateral IH, the same procedure was repeated on the contralateral side, ensuring a 1-2-cm overlap of the mesh medially. The port sites were closed with skin staplers.

\section{Post-operative Management}

Injection paracetamol $1 \mathrm{~g} 8$ hourly was given to all patients on the day of surgery for analgesia. Oral fluids were allowed $6 \mathrm{~h}$ post-operative and progressed to normal diet the next day. Visual analog scale was used to assess pain in the post-op period.

\section{Follow Up}

The patients were regularly followed up. Follow-up was done at 1 week, 3 months, 6 months, and 1 year. All patients completed a minimum of 1 year follow-up and we could achieve nearly $96 \%$ clinical follow-up at the end of 1 year of surgery. The follow-up data for those patients who subsequently did not report for further follow-up was obtained by the means of telephonic interview.

\section{Statistical Analysis}

Statistical analysis was done using the statistical software Graphpad available at www.graphpad.com. Continuous data are presented as mean \pm standard deviation and categorical data as numbers and percentages. Categorical variables were compared using chi-square test or Fisher's exact test whichever applicable. Continuous variables were compared using 
Fig. 1 Flow chart of patients and the follow-up

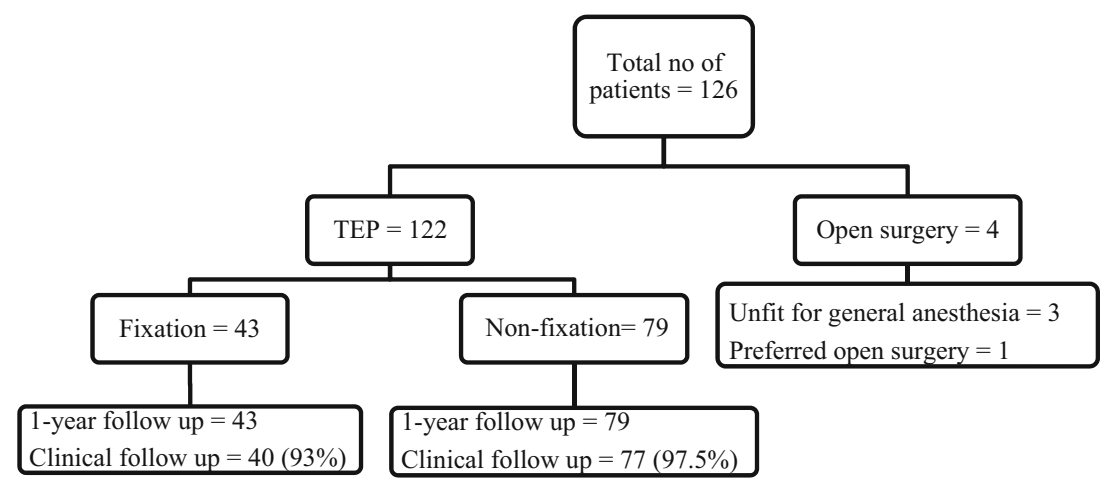

Student's $t$ test. A $p$ value of $<0.05$ was considered as statistically significant.

\section{Results}

During this period, $175 \mathrm{IH}$ repairs were done in126 patients at our department. One hundred twenty-two patients underwent laparoscopic and 04 underwent open IH surgery. Till June 2013, 43 patients underwent TEP and all underwent mesh fixation. Thereafter, 79 patients underwent TEP in which the mesh was not fixed (Fig. 1).

\section{Patient Demographics}

Patient characteristics and their clinical profile are given in Table 1 . The mean age of the patient was $49.4 \pm 19.9$ and $47.1 \pm 13.8$ years in the fixation and non-fixation group, respectively. Expectedly, the vast majority of the patients were males.

Table 1 Patient demographics and characteristics of hernia

\begin{tabular}{lll}
\hline Variables & $\begin{array}{l}\text { Fixation } \\
(n=43)\end{array}$ & $\begin{array}{l}\text { Non-fixation } \\
(n=79)\end{array}$ \\
\hline Age (years) & & \\
Mean \pm SD (range) & $49.4 \pm 19.9(18-73)$ & $47.1 \pm 13.8(21-79)$ \\
Male (\%) & $42(97.7)$ & $76(96.2)$ \\
ASA grade (\%) & & $49(62)$ \\
ASA I & $29(67.4)$ & $30(38)$ \\
ASA II & $14(32.6)$ & \\
Duration of symptoms & & $18.2 \pm 7.2$ \\
$\quad($ months) & & $73(92.4 \%)$ \\
Mean \pm SD & $22.1 \pm 9.7$ & $6(7.6 \%)$ \\
Primary hernias $(\%)$ & $39(90.7 \%)$ & $46(58.2 \%)$ \\
Recurrent hernias $(\%)$ & $4(9.3 \%)$ & 33 \\
Unilateral $(\%)$ & $27(62.8 \%)$ & 13 \\
Right & 20 & $33(41.8 \%)$ \\
Left & 07 & 112 \\
Bilateral $(\%)$ & $16(37.2 \%)$ & \\
Number of repairs & 59 & \\
\hline
\end{tabular}

\section{Hernia Characteristics}

In all, 171 TEP repairs were done in 122patients. Of the 122 patients, 43 were in the fixation group and 79 in the nonfixation group. In the fixation group, there were 16 bilateral hernias and 33 bilateral hernias in the non-fixation group. Thus, the total number of repairs was 59 and 112 in the two groups, respectively (Table 1).

\section{Operative Times}

The mean operative times for unilateral $\mathrm{IH}$ for the fixation and non-fixation groups were $41.8 \pm 11.4$ and $35.9 \pm 9.7 \mathrm{~min}$, respectively. This was statistically significant $(p=0.021)$. The same for bilateral hernias, operative times were $66.2 \pm 15.6$ and $55.3 \pm 14.2 \mathrm{~min}$, respectively, for the two groups. This too was statistically significant $(p=0.018)$. The intraoperative factors and complications are given in Table 2.

\section{Post-operative Pain}

The pain score was evaluated at $24 \mathrm{~h}$ post-op using a visual analog scale. The mean pain score was significantly more in the fixation group, $3.44 \pm 1.2$ versus $3.01 \pm 1.0$ in the two groups, respectively $(p=0.037)$.

\section{Hospital Stay and Return to Normal Activity}

The hospital stay and days taken to return to normal activity were similar in both groups. The other post-op complications are given in Table 3.

\section{Chronic Groin Pain and Hernia Recurrence}

The overall incidence of CGP was not different in the two groups. The incidence of CGP was $02(3.4 \%)$ and $3(2.7 \%)$ in the fixation and non-fixation groups, respectively, which was not statistically significant $(p=1.000)$. Further, there was no increased incidence of recurrences in the non-fixation group. At a mean follow-up of $33.2 \pm 17.0$ and $18.7 \pm 6.2$ months, there were 
Table 2 Intra-operative factors and complications

\begin{tabular}{llll}
\hline & Fixation & Non-fixation & $p$ value \\
\hline $\begin{array}{l}\text { Mean operative time } \\
\text { (minutes) }\end{array}$ & & & \\
Unilateral & $41.8 \pm 11.4$ & $35.9 \pm 9.7$ & 0.021 \\
Bilateral & $66.2 \pm 15.6$ & $55.3 \pm 14.2$ & 0.018 \\
Conversion & $02(4.6 \%)$ & 0 & 0.122 \\
Injury to viscera & Nil & Nil & - \\
Injury to vas deferens & Nil & Nil & - \\
Injury to testicular vessels & $01(1.7 \%)$ & $2(1.8 \%)$ & 1.0 \\
Injury to inferior epigastric vessels & $01(1.7 \%)$ & $4(3.6 \%)$ & 0.667 \\
Injury to major vessels & Nil & Nil & - \\
Extensive surgical emphysema & $01(2.3 \%)$ & $2(2.5 \%)$ & 1.0 \\
\hline
\end{tabular}

$02(3.4 \%)$ recurrences in the fixation group while there were none in the non-fixation group, respectively $(p=0.118)$ (Table 4).

\section{Discussion}

Today, the major issue with IH surgery is the incidence of CGP along with recurrence rates. During LIHS, mesh fixation is done routinely in order to prevent mesh migration which may lead to uncovering of the defect and, consequently, increased recurrences. It has been shown that mesh fixation with tackers/sutures can lead to increased incidences of both acute and chronic groin pain $[13,14]$. Since CGP majorly affects the QOL of patients undergoing IH surgery, concerns have been drawn towards mesh fixation. However, the opposing view is that if mesh fixation was not done, it would lead to increased recurrences. Three meta-analyses have looked at this issue and have comprehensively concluded that the recurrence rates are not increased by non-fixation of the mesh [10-12].

This retrospective study undertaken at the gastrointestinal and minimally invasive surgery center of a tertiary care hospital looking into the merits of non-fixation of mesh found that the recurrence rates were not increased but the incidence of CGP was not different from the fixation group. We achieved a minimum of 1-year follow-up for all patients and close to $96 \%$ clinical follow-up. A consensus meeting recommended that for recurrence, reporting only a clinical follow-up will be valid [15]. The other significant differences found were shorter operative times and decreased pain scores in the early post-op period in the non-fixation group as compared to the fixation group.

It has been postulated that placement of tacks can damage the nerves directly or indirectly, by fibrosis around the tack which can go on to involve the nerves [16]. The chances of nerve damage go up with the number of tacks being used. Consequently, the practice now is to apply tacks medially in the region of Cooper's ligament and laterally at the level of ASIS [9]. These areas are far away from the nerves. CGP was assessed as defined by International Association of the Study for Pain: Pain persisting beyond the normal tissue healing time assumed to be 3 months [17]. There was no difference in the incidence of CGP in the two groups. This was probably due the surgeons' experience who avoided nerve damage by
Table 3 Post-operative factors and complications

\begin{tabular}{llll}
\hline & Fixation & Non-fixation & $p$ value \\
\hline Pain score at 24h & $3.44 \pm 1.2$ & $3.01 \pm 1.0$ & 0.037 \\
Urinary retention & $02(4.6 \%)$ & $6(7.5 \%)$ & 0.713 \\
Seroma at 1 week & $04(6.8 \%)$ & $5(4.5 \%)$ & 0.723 \\
Funiculitis & $01(1.7 \%)$ & $1(0.9 \%)$ & 1.0 \\
Ischemic orchitis & Nil & Nil & - \\
Ecchymosis & $01(1.7 \%)$ & $3(2.7 \%)$ & 1.0 \\
Wound infections & Nil & Nil & - \\
Hospital stay (days) & $1.42 \pm 0.7$ & $1.36 \pm 0.8$ & 0.680 \\
Mean \pm SD & & & 0.897 \\
Days to return to normal activity & $7.91 \pm 2.1$ & $7.96 \pm 2.0$ & \\
\hline
\end{tabular}


Table 4 Follow-up and short-term outcomes

\begin{tabular}{llll}
\hline & Fixation & Non-fixation & $p$ value \\
\hline Mean follow-up (months) & $33.2 \pm 17.0$ & $18.7 \pm 6.2$ & \\
Mesh infection & $1(1.7 \%)$ & 0 & 0.357 \\
Chronic groin pain & $02(3.4 \%)$ & $3(2.7 \%)$ & 1.000 \\
Port site hernia & Nil & Nil & - \\
Recurrence & $02(3.4 \%)$ & 0 & 0.118 \\
\hline
\end{tabular}

meticulous dissection and precise placement of tacks. It is recommended that additional tack if used should be placed above the iliopubic tract as the nerves are below this level. However, it has been shown that in up to $15 \%$ of cases, the nerves may course above the level of the tract.

A very significant finding in this study was that the nonfixation of mesh did not lead to increased recurrence. This is in agreement to the results of randomized trials and metaanalysis looking at non-fixation of mesh [10-12]. A few studies have used preformed or 3-D meshes to prevent mesh slippage $[14,18]$. However, in our study, we did not use any preformed meshes and did not find increased recurrences.

It has been reported by a few studies that use of tacks for fixing mesh leads to increased post-op pain $[14,18]$. Our study too found increased scores in the fixation group when we assessed pain at $24 \mathrm{~h}$ post-surgery and this was statistically significant. However, the hospital stay as well as the time taken to return to normal activity was similar in the two groups. A consequence of increased pain in the immediate post-op period is the increased incidence of urinary retention which has been reported by a few studies [14, 18, 19]. The overall incidence of urinary retention was similar to reported literature but we did not find an increased incidence in the fixation group.

Fixation of mesh leads to increased operative time. This has been reported by many authors [20] while the most recent meta-analysis did not find a statistically significant difference in operating times in the two groups [12]. Our study revealed that fixation of mesh indeed leads to significantly increased operative times and was found in both unilateral and bilateral IH repairs.

The use of laparoscopic tackers would entail additional costs. Three trials have shown that non-fixation of mesh leads to decreased costs [19-21]. One randomized trial reported that the difference in the two groups was \$120 [19]. Ours is a government-funded hospital and as such estimating costs to the patient is difficult to assess accurately. An endoscopic tacker costs around rupees 20,000. Since the procedure remains the same except for the use of tacker, we can assume that this would be the difference in the cost notwithstanding the cost saved due to lesser operating times. A concern about LIHS has been that it is costlier than open IH surgery. In our study, in addition to not using tackers, we have avoided using dissecting balloon to create preperitoneal space and a preformed mesh. This would significantly bring down costs of LIHS and could match the cost of open IH repair [19].

Indirect advantages of non-fixation of mesh are that one need not worry about the injuries to the vascular structures and nerves in the preperitoneal space. As Lap TEP has got a steep learning curve, there are less chances of vascular/nerve injuries by the junior surgeons when mesh fixation is not done. Another point to be made is that in the rare event of a mesh infection, the mesh can be extracted easily and comes out in toto.

Our study has some limitations. It is a retrospective study and the fixation group will be akin to a historical control. As is well known, the results of a given surgery tend to improve over time. Thus, we have no conversions and recurrences in the non-fixation group. Also, evident is a longer follow-up in the fixation group. Notwithstanding this, all patients were followed up for a minimum of 1 year and almost all were clinical follow-ups. Since most recurrences occur within the first year post-surgery, we would not consider this as a major issue.

\section{Conclusion}

This study reveals that avoidance of fixation of mesh during totally extra peritoneal repair of inguinal hernias is as safe as mesh fixation with certain advantages. It does not lead to increased recurrence though it does not decrease the incidence of chronic groin pain. Collateral advantage would be decreased operative times, lesser post-operative pain, and decreased costs.

\section{Compliance with Ethical Standards}

Conflict of Interest The authors declare that they have no conflict of interest.

\section{References}

1. NICE Guidelines (2007) Laparoscopic surgery for inguinal hernia repair. Technology appraisal guidance 83 www.nice.org.uk

2. Arregui MR, Davis CD, Yucel O, Nagan RF (1992) Laparoscopic mesh repair of inguinal hernia using a preperitoneal approach: a preliminary report. Surg Laparosc Endosc 2(1):53-58

3. Dulucq JL (1991) Traitement des hernies de l'aine par la mise en place d'un patch prothetique par laparoscopie. Voitotalementextraperitoneale. CahChir 79:15-16

4. Kockerling F, Schug-Pass C, Jacob DA, Keller T (2013) The intraand postoperative complication rate of TEP in patients undergoing unilateral endoscopic inguinal hernia repair is not higher compared with TAPP. World J Surg 37(4):933-934. https://doi.org/10.1007/ s00268-012-1858-8 
5. Singh AN, Bansal VK, Misra MC, Kumar S, Rajeshwari S, Kumar A et al (2012) Testicular functions, chronic groin pain and quality of life after laparoscopic and open mesh repair of inguinal hernia: a prospective randomized controlled trial. Surg Endosc 6(5):1304-1317

6. Jakhmola CK, Kumar A (2015) Laparoscopic inguinal hernia repair in the armed forces: a 5 year single centre study. MJAFI 71(4):317-323

7. Amid PK, Shulman AG, Lichtenstein IL (1996) Open "tensionfree" repair of inguinal hernias: the Lichtenstein technique. Eur J Surg 162(6):447-453

8. Koninger J, Redecke J, Butters M (2004). Chronic pain after hernia repair: a randomized trial comparing Shouldice, Lichtenstein and TAPP. Laangenbecks Arch Surg 389(5):361-365, DOI: https://doi. org/10.1007/s00423-004-0496-5

9. Felix E, Scott S, Crafton B, Geis P, Duncan T, Sewell R, McKernan B (1998) Causes of recurrence after laparoscopic hernioplasty. A multicenter study. Surg Endosc 12(3):226-231. https://doi.org/10. 1007/s004649900640

10. Teng YJ, Pan SM, Liu YL, Yang KH, Zhang YC, Tian JH et al (2011) A meta-analysis of randomized controlled trials of fixation versus nonfixation of mesh in laparoscopic total extraperitoneal inguinal hernia repair. Surg Endosc 25:2849e 58

11. Tam KW, Liang HH, Chai CY (2010) Outcomes of staple fixation of mesh versus nonfixation in laparoscopic total extraperitoneal inguinal repair: a metanalysis of randomized controlled trials. World J Surg 34:3065e 74

12. Sajid MS, Ladwa N, Kalra L, Hutson K, Sains P, Baig MK (2012) A meta-analysis examining the use of tacker fixation versus nofixation of mesh in laparoscopic inguinal hernia repair. Int J Surg 10(5):224-231. https://doi.org/10.1016/j.ijsu.2012.03.001

13. Lau H, Patil NG (2004) Acute pain after endoscopic totally extraperitoneal (TEP) inguinal hernioplasty: a multivariate analysis of predictive factors. Surg Endosc 18(1):92-96. https://doi.org/10. 1007/s00464-003-9068-y
14. Koch CA, Greenlee SM, Larson DR, Harrington JR, Farley DR (2006) Randomized prospective study of totally extraperitoneal inguinal hernia repair: fixation versus no fixation of mesh. JSLS 10(4):457-460

15. Muysoms FE, Deerberg EB, Peeters E, Agresta F, Berrevoet F, Campanelli G et al (2013) Recommendations for reporting results in abdominal wall repair: results of a consensus meeting in Palermo, Italy, 28-30 June 2012. Hernia 17(4):423-433

16. Stark E, Oestreich K, Wendl K, Rumstadt B, Hagmüller E (1999) Nerve irritation after laparoscopic hernia repair. Surg Endosc 13(9): 878-881. https://doi.org/10.1007/s004649901124

17. International association for the study of pain (1986) Subcommittee on Taxonomy. Classification of chronic pain: description of chronic pain syndromes and definition of pain terms. Pain 3:S1-226

18. Li JW, Zheng MH, Li HQ, Zhang H, Hu WG, Wang ML (2007) A randomized controlledclinical trial comparing stapling with nonstapling of mesh in laparoscopic total extraperitoneal inguinal hernioplasty. Chin J Gen Surg 22:440-442

19. Ferzli GS, Frezza EE, Pecoraro AM Jr et al (1999) Prospective randomized study of stapled versus unstapled mesh in a laparoscopic preperitoneal inguinal hernia repair. J Am Coll Surg 188(5):461465. https://doi.org/10.1016/S1072-7515(99)00039-3

20. Tam KW, Liang HH, Chai CY (2010) Outcomes of staple fixation of mesh versus nonfixation in laparoscopic total extraperitoneal inguinal repair: a metanalysis of randomized controlled trials. World J Surg 34(12):3065-3074. https://doi.org/10.1007/s00268010-0760-5

21. Moreno-Egea A, Torralba Martinez JA, Morales Cuenca G et al (2004) Randomized clinical trial of fixation vs nonfixation of mesh in total extraperitoneal inguinal hernioplasty. Arch Surg 139(12): 1376-1379. https://doi.org/10.1001/archsurg.139.12.1376 